\title{
Registro del ingreso de MADERA de pino en ARgentinA, A PARTIR DE CONSTRUCCIONES HISTÓRICAS
}

\author{
A. M. GIMÉNEZ¹, J. GÓMEZ², J. G. MOGLIA¹, J. DÍAZ ZÍRPOLO¹ y D. GONZALEZ
}

\begin{abstract}
Summary: Record of the entry of pine wood in Argentina from historical buildings. The objective of this work is to analyze under what period was introduced pine wood from historical buildings in Argentina. The work is based on characterizing and determining wooden pieces of historical constructions. Were analyzed woods belonging to: The Pioneers Museum (Río Gallegos, Santa Cruz), Church of Our Lady of the Rosary (Salsipuedes, Córdoba) and Céspedes Tower (Jesus María, Córdoba). Ten samples were analyzed corresponding to wooden roof structures. Terminology of the Nomenclature Committee of IAWA for characterization of wood was followed. The timber identification keys that were used are: Jacquiot; Clement, Greguss and García Esteban et al. Pinus woods were used in the three analyzed buildings. These buildings date from the late nineteenth century and certified the importation of gymnosperm wood, from the middle of this century. These references allow discerning the constructive habits. From these studies, we can infer changes in the use of wood in the country and opening up to European and American markets.
\end{abstract}

Key words: Wood, historic, determination, Pinus.

Resumen: Es objetivo del trabajo analizar, a partir de construcciones históricas, en qué periodo fue introducida la madera de pino en Argentina. El trabajo se basa en caracterizar y determinar piezas de madera de construcciones históricas. Se estudiaron muestras de maderas pertenecientes a: Museo de Los Pioneros (Río Gallegos, Santa Cruz); Iglesia Nuestra Señora del Rosario (Salsipuedes, Córdoba) y Torre de Céspedes (Jesús María, Córdoba). Fueron analizadas diez muestras de madera correspondientes a las estructuras del techo. Se siguió la terminología del Comité de Nomenclatura de IAWA para la caracterización de la madera. Se utilizaron las Claves de identificación de madera de los siguientes autores: Jacquiot; Clement, Greguss y García Esteban et al. Se observó que en los tres edificios históricos fue empleada madera de diferentes especies de pino. Las construcciones datan de fines del siglo XIX y certifican la importación de madera de gimnospermas, a partir de mediados de dicho siglo. Estas referencias permiten discernir sobre las costumbres constructivas. A partir de estos estudios, se pueden inferir los cambios en el empleo de la madera en el país y la apertura a los mercados europeos y americanos.

Palabras clave: Madera, histórica, determinación, pino.

\section{INTRODUCCIÓN}

La madera es un material biológico heterogéneo, anisotrópico e higroscópico, presente abundantemente en la naturaleza (Giménez \& Moglia, 1999). Presenta amplia versatilidad de respuestas frente a los factores medioambientales, superior a la mayor parte de los materiales empleados en la construcción.

${ }^{1}$ Facultad de Ciencias Forestales, UNSE, Avda. Belgrano 1912 (s) 4200. Santiago del Estero. 2- Facultad de Arquitectura. UNC. Córdoba. Email: amig@unse.edu.ar
Requiere de menor gasto energético en su producción, es natural, biodegradable, reciclable, buen aislante no tóxico, además fija $\mathrm{CO}_{2}$ durante su crecimiento (Romero, 2014). Ha sido el recurso de mayor empleo como material en la construcción, desde la prehistoria hasta principios de la era industrial. En Argentina la madera se ha usado desde tiempos remotos. En primera instancia, en la época precolombina y colonial, se emplearon maderas nativas, posteriormente, a partir de 1850, se comenzaron a importar maderas para la construcción y el uso estructural, especialmente de Gimnospermas (Evans et al., 2008). 
Las maderas de coníferas de alta calidad, codiciadas y distribuidas por el mundo para diferentes usos, son principalmente las pertenecientes a especies de crecimiento más lento de las zonas frías, como las de los géneros Abies, Picea, Juniperus, Larix, Tsuga y Pseudotsuga y pinos (Picea abies Karts., Pinus montícola Dougl., P. nigra Arn., P. taeda L., P. ponderosa Laws., etc.), característicos de Escandinavia, Europa central, Canadá y Estados Unidos (Greguss, 1955). Sus usos más destacados son para pastas celulósicas de alta calidad y como madera de uso estructural -para construcción, aberturas, carpintería y muebles-. La estructura celular homogénea de la madera de coníferas, formada por traqueidas largas y uniformes, se traduce en alta resistencia mecánica (en particular al tener en cuenta su densidad), a la vez que le confiere flexibilidad y capacidad para soportar grandes esfuerzos (SEC \& SPC, 2001).

Éstas maderas cuentan con creciente popularidad basada en las ventajas que ofrece a los usuarios. Dentro de las ventajas se destacan las medidas y clases resistentes normalizadas; el control de calidad, a través del cumplimiento de un único y unificado sistema de clasificación; la resistencia y durabilidad; la posibilidad de aplicación de tratamientos para su protección contra agentes xilófagos y contra el fuego; y la sistematización y normalización para la construcción. Además, debido a su inherente versatilidad y gran adaptabilidad, la mayoría de ellas se prestan a diferentes adaptaciones tecnológicas y pueden combinarse con materiales plásticos para obtener una gran variedad de laminados y chapas (AITIM, 2008).

Desde el siglo XIX, con la llegada de importantes cantidades de barcos mercantes con el objetivo de recoger carne y otros productos agropecuarios, llegan las primeras partidas de éstas maderas al mercado argentino. En efecto, dichos barcos llegaban al país lastrados con madera de pinotea. MaderSa de excelente calidad, presente en abundancia, se convirtió en un material muy importante para la construcción de viviendas, integrándose a la construcción tradicional (Evans et al., 2008).

Con el apelativo pinotea se hace referencia a diferentes especies de los géneros Pseudotsuga y
Pinus; generalmente eran importados de Estados Unidos. Estas maderas se caracterizan por presentar anillos de crecimiento muy delgados, alta proporción de leño tardío y abundantes canales de resina, lo que configura un material sólido, durable y de mayor densidad básica que los pinos de rápido crecimiento (García Esteban et al. 1996).

Entre las tradiciones ancestrales, la utilización de la madera como material se ha basado en el conocimiento de sus comportamientos para alcanzar excelentes resultados. En muchas oportunidades han podido sobrevivir en buenas condiciones durante miles de años a pesar de su fragilidad. La práctica ha permitido saber que, para el mejor rendimiento de la madera, hay que ser cuidadoso en su corte, en las condiciones de almacenamiento y secado, también con los diferentes tratamientos a los que se la puede someter para mejorarla (Johnson, 1989). En las épocas modernas y contemporáneas, la madera sigue siendo la materia prima esencial en los campos de la producción, de la construcción de edificios, de mobiliario y decoraciones.

Parte de la historia de los pueblos, puede ser interpretada a través de los usos de la madera. Los estudios de identificación de maderas de objeto y obras patrimoniales han aportado datos muy importantes para estudios arqueológicos, históricos y etnobotánicos (Bauch \& Eckstein, 1981; Carreras \& Deschamps, 1995; Giménez et al., 2012).

Rodríguez Trobaj (2008) trató aspectos significativos del ciclo constructivo de las maderas de pino silvestre/laricio en edificios históricos de España, proporcionando nuevos elementos de discusión acerca de la historia cultural y natural del material leñoso. Como abordaje, se utiliza el método empírico dendrocronológico para la datación de madera, pero al mismo tiempo como instrumento de análisis ecofactual (identificación y procedencia) y artefactual (uso constructivo y deterioro). Se parte de la identificación taxonómica y cronológica del material para llegar a establecer las zonas geográficas de procedencia. La procedencia del material es una cuestión básica del análisis arqueológico de una construcción ya que el acceso y abundancia del recurso condiciona su utilización y el desarrollo de modos y tipos constructivos determinados. 
La importancia de las construcciones históricas simbolizando la identidad nacional de un país, obliga a los profesionales a buscar técnicas científicas que permitan inspeccionarlas sin intervenciones que perjudiquen la edificación (Mantilla Carrasco et al., 2009). En la carpintería de estructuras edilicias históricas argentinas, un aspecto importante a determinar es la distancia de transporte del material de construcción y la adaptación al recurso leñoso que refleja la utilización de fuentes de aprovechamiento múltiples, de manera que es frecuente encontrar en la misma construcción varias especies de maderas (Giménez et al., 2014). El trabajo de identificación de maderas de objetos de significancia histórica requiere de una acción combinada entre los gestores del patrimonio histórico y especialistas en maderas.

El objetivo del trabajo es, a través de la caracterización e identificación de piezas de madera de construcciones históricas de fines del siglo XIX, analizar los primeros ingresos de maderas de coníferas a la Argentina e identificar las especies y su procedencia.

\section{Material y Métodos}

Desde el año 2005, el LAM (Laboratorio de Anatomía de Maderas) del Instituto de Silvicultura y Manejo de Bosques (INSIMA),
Facultad de Ciencias Forestales, UNSE, trabaja en conjunto con Facultad de Arquitectura UNC bajo la dirección del Ing. José Gómez, en un proyecto de cooperación interinstitucional para la Recuperación del patrimonio histórico analizando sistemas estructurales constructivos. En el presente trabajo se tratarán 3 construcciones que datan de fines del siglo XIX.

1 - Parroquia Nuestra Señora del Rosario, Salsipuedes, Córdoba (Fig. 1). En 1801 es la primera mención sobre la existencia del recinto sagrado de Salsipuedes. La actual Capilla, con una arquitectura sencilla y austera, data de 1875/1885. En 1894 se rehízo el techo y se determinó el sistema a dos aguas, tal como hoy se conserva. Se analizaron 2 piezas de madera estructural: Muestra 1- Cordón superior; Muestra- 2 Cordón inferior.

\section{2 - Casa Parisi, Museo de los Pioneros,} Municipalidad de Río Gallegos (Fig. 2).

Los habitantes de la ciudad de Río Gallegos reconocen la Casa Parisi como una de las pocas que sobrevivieron los duros inviernos y largas épocas de viento que azotan el extremo sur argentino. En ella funciona el Museo de los Pioneros y es un fantástico viaje hacia los primeros años del pueblo. Entre 1892 y 1897, los hermanos Fenton le cedieron el espacio al gobernador del territorio de Santa Cruz Edelmiro Mayer, al quedarse este sin vivienda por un incendio de la sede gubernamental.

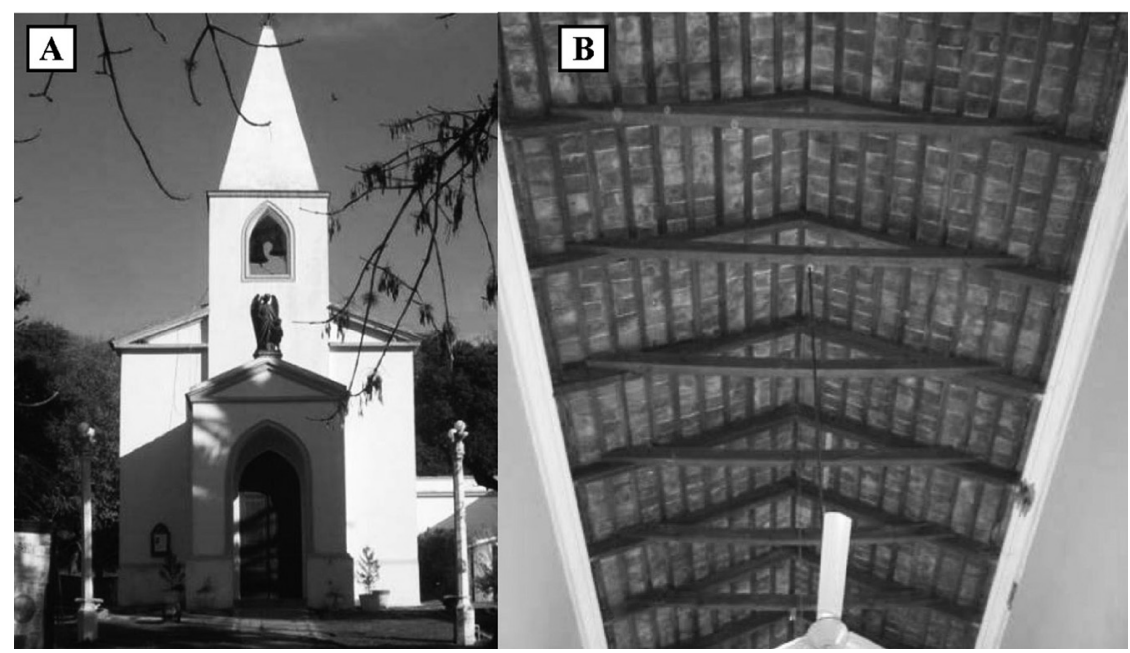

Fig. 1. Iglesia Nuestra Señora del Rosario, Salsipuedes, Córdoba. A: Exterior. B: Interior. 


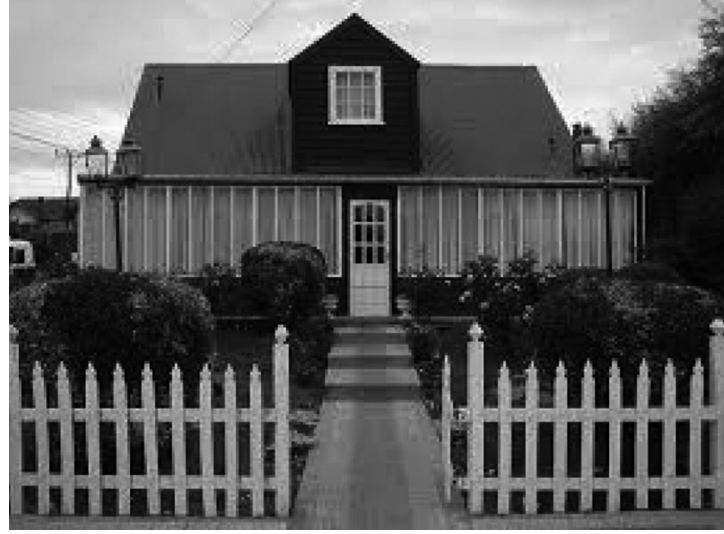

Fig. 2. Fachada de la casa Parisi, Museo de los Pioneros, Municipalidad de Rio Gallegos.

Posteriormente la casa fue habitada por Roque Parisi y su familia, y de allí el nombre con que se la conoce actualmente. La señora Parisi habilitaba un sector del patio de su casa para que los paisanos sureños que llegaban al pueblo pudieran armar sus toldos y guarecerse. El municipio de Río Gallegos recuperó la casa más antigua en pie de la ciudad, organizó el museo para testimoniar la vida de los pioneros a través de los enseres que sus familiares cedieron en préstamo. El Museo de Los Pioneros está tratando de rescatar la historia de la casa, en cuyo origen hay confusión. Los primeros pobladores de la zona fueron de origen británico. Se cree que la casa fue traída de Europa y armada en 1890. Lo que sí es comprobable es que en sus viejas maderas perduran las iniciales de los que la armaron: Greenshilds, Redman y Woodman, quienes componían una sociedad ganadera de la época y figuran como extranjeros con tenencia de tierras en Santa Cruz $(1893,1920)$. La arquitectura responde al tipo de construcción inglesa (Secretaría de Turismo, 2014).

Las piezas para su estudio son 2: Muestra 1 tabla de piso en planta alta, Muestra 2- tablas del revestimiento exterior.

\section{3 - Torre de Céspedes de Jesús María, provincia de Córdoba (Fig. 3).}

La construcción de Gabriel Céspedes data de fines del siglo XIX. Con rasgos característicos de la arquitectura española, fue edificada como casa de veraneo en 1896. El diseño del castillo

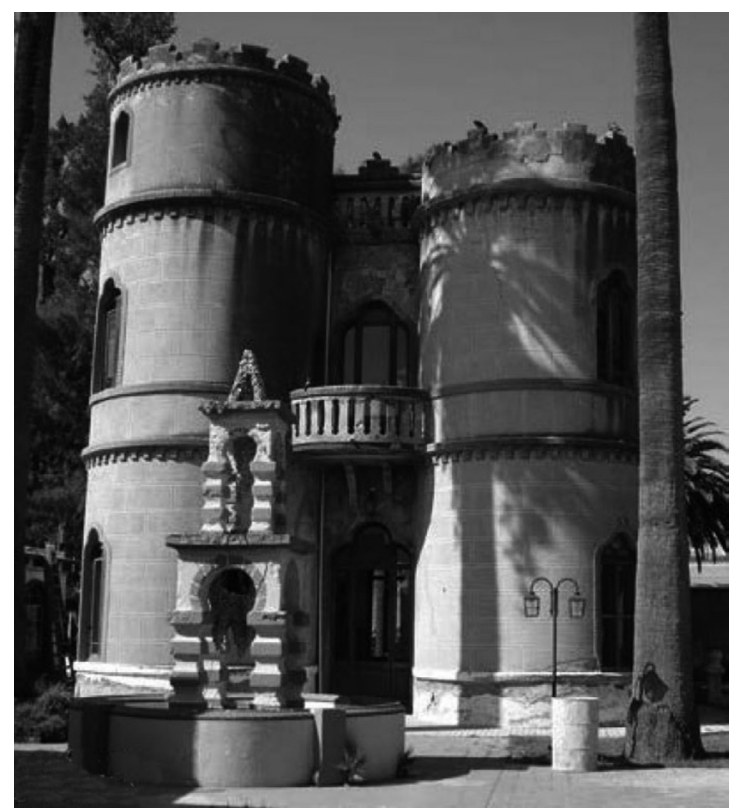

Fig. 3. Torre Céspedes, Jesús María, Córdoba.

corresponde a Pedro Buscá, quien esbozó un octógono central y cuatro torres circulares, dividido en dos plantas. En 1995 la ordenanza 1713 declaró de Interés Municipal y componente del Patrimonio Arquitectónico Urbanístico de Jesús María esta obra, que agrupa detalles de estilo europeo. En el proceso de restauración a nivel subterráneo se descubrió parte de la antigua acequia abovedada que pasaba por el predio, elemento de realce como construcción histórica (Gómez \& Ruata, 2009).

Se identificaron de 2 muestras de madera: Muestra 1- Piso de la Torre; Muestra 2- Viga principal de la escalera helicoidal de la torre.

El trabajo de determinación de maderas puestas en obra en edificios históricos, requiere de una extracción cuidadosa cuando se emplean métodos destructivos de determinación. Las muestras deben ser lo suficientemente grandes para poder realizar los cortes a los distintos planos y lo suficientemente pequeñas para no dañar el objeto de trabajo. Cada material fue analizado macro y microscópicamente para su identificación. Para la caracterización macroscópica se empleó lupa estereoscópica x 100, 160 aumentos; para la microscópica, microscopio óptico Zeiss con videocámara. Fueron cortadas con micrótomo 
de carro móvil Leitz en plano transversal y longitudinal tangencial y radial. En algunos casos las muestras fueron teñidas con coloración crisoidina-acridina roja (Freund, 1970). En otras situaciones se realizaron preparados temporarios. En las descripciones se siguió la terminología del Comité de Nomenclatura de IAWA (Ritcher et al., 2004) y Tortorelli (2009). Se consignan algunos caracteres anatómicos que hacen a la determinación de la especie. Se utilizaron las Claves de identificación de madera: Tortorelli (2009), Jacquiot (1955), Greguss (1955) y García Esteban et al. (1996).

\section{Resultados y Discusión}

\section{1 - Iglesia de Salsipuedes}

\section{Caracterización anatómica}

Análisis macroscópico: madera castaño amarillento (Fig. 4A), anillos muy demarcados, con neta diferenciación leño temprano/tardío, el espesor medio de anillos es de $5 \mathrm{~mm}$ (Fig. 4B). Anillos de ancho irregular, con leño tardío de más de 20 hileras de células (Fig. 4C). En algunos anillos corresponde al leño tardío más del $70 \%$. Hay falsos anillos. Madera en muy buen estado por la gran cantidad de sustancias resinosas.

Análisis microscópico: leño traqueidal, anillos de crecimiento muy demarcados, con transición abrupta entre leño temprano/tardío, con canales de resina axiales de paredes delgadas y dimensión media. Traqueidas axiales de paredes gruesas con puntuaciones uniseriadas, en menor proporción biseriadas opuestas en las paredes radiales, con crásulas (Fig. 4D). Canales resiníferos axiales escasos de paredes delgadas, básicamente en el leño tardío. Parénquima axial ausente. Radios uniseriados heterocelulares, de altura máxima 1015 células; puntuaciones del campo de cruzamiento pinoides (1-3 por campo) (Fig. 4E); traqueidas radiales marginales (1-2) e internas con identaduras (Fig. 4F). Canales secretores radiales pequeños en algunos radios (Fig. 4G). Anomalías en la proporción de leño temprano a tardío.

Por las características de la madera se indica que corresponde M1 y M2 a: Gimnosperma, Familia Pinaceae, género Pinus, especie: Pinus taeda L.

La madera de Pinus taeda (conocida vulgarmente como pinotea), proviene de Estados Unidos, el área de dispersión natural, donde el crecimiento es muy inferior al que actualmente se obtiene de plantaciones comerciales de Argentina. Por esta razón la madera de origen norteamericano presenta mayor peso específico y mayores valores de resistencia. Es interesante comprobar que en la época de la construcción de la iglesia se importaba madera norteamericana para las construcciones civiles y religiosas. Posiblemente la causa de ello, es la disponibilidad de madera de largas dimensiones y de excelente resistencia, y bajo peso (Gimnospermas), ideal para estructuras.

\section{2 - Museo Parisi. (Fig. 5)}

M1- Madera en muy buen estado por la gran cantidad de sustancias resinosas. Leño traqueidal, anillos de crecimiento muy demarcados (Fig. $5 \mathrm{~A})$, con transición abrupta entre leño temprano y tardío (Fig. 5B). Traqueidas axiales con puntuaciones uniseriadas y a veces biseriadas en las paredes radiales, se observan crásulas (Fig. 5C). Canales resiníferos axiales escasos de paredes delgadas, básicamente en el leño tardío. Radios uniseriados heterocelulares, de altura máxima 1015 células. Puntuaciones del campo de cruzamiento fenestriformes con 1-2 por campo (Fig. 5D), se destaca la presencia de traqueidas radiales marginales (1-2) e internas, con identaduras poco pronunciadas. Parénquima axial ausente. Canales de resina axiales de paredes delgadas y dimensión media, canales horizontales pequeños en algunos radios.

Por las características de la madera se indica que corresponde a: Gimnosperma, Familia Pinaceae, Género Pinus, Sección Sylvestris, especie: Pinus sylvestris $\mathrm{L}$.

Especie de distribución muy amplia, origen europeo y asiático. Madera resinosa de buena calidad que produce mayor proporción de duramen y albura más colorada en las variedades de mejor porte y albura más blanca y gruesa en las de peor porte (García Esteban et al. 1996). La madera es en general de buena calidad, con troncos rectos y poco nudosos, propiedades que han conseguido que sea uno de los pinos más apreciado. Por su calidad y su dureza media, se utiliza en construcción, ebanistería y carpintería, muebles de calidad y para mástiles de barcos. 
Bol. Soc. Argent. Bot. 49 (4) 2014
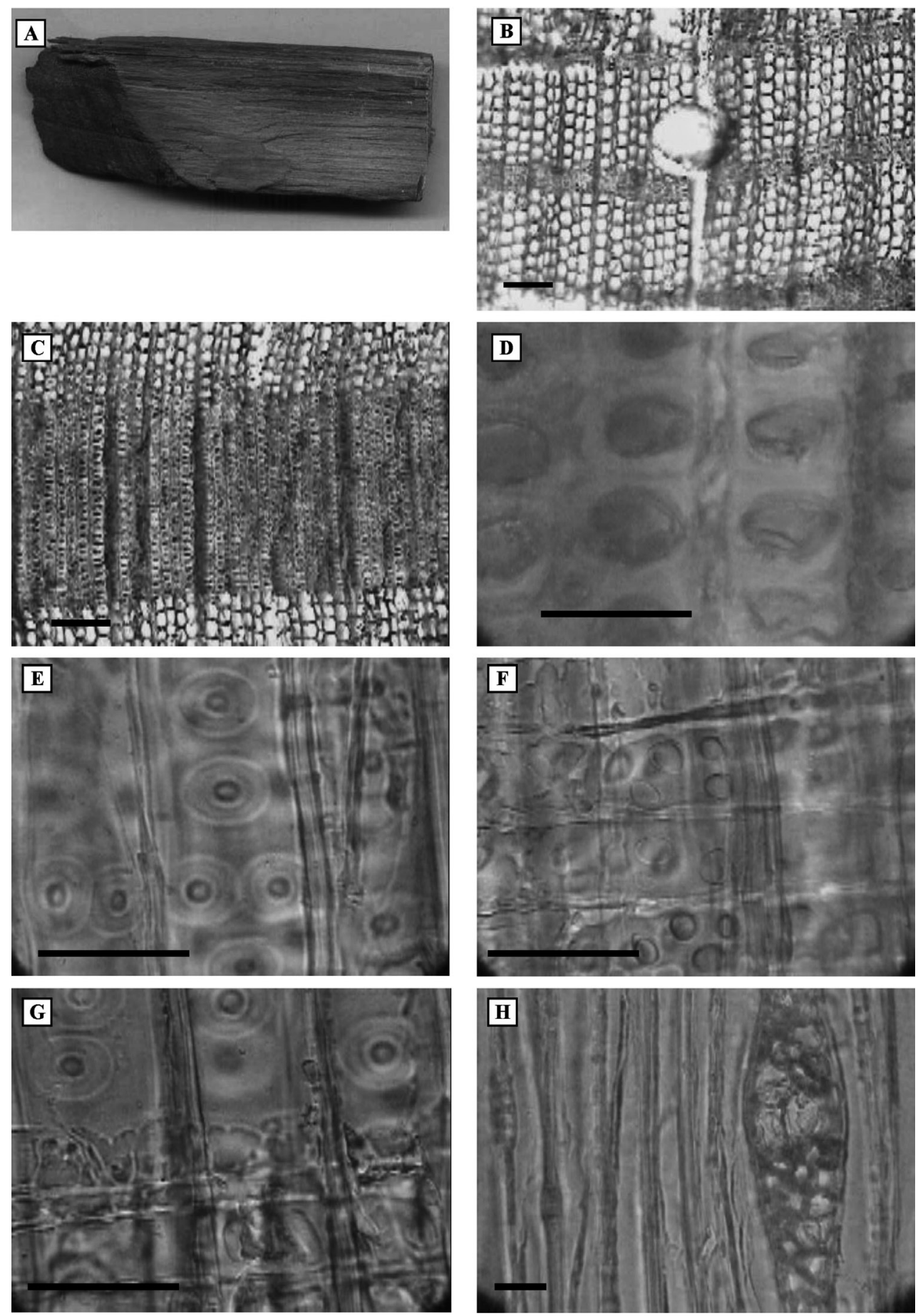

Fig. 4. Pinus taeda L. A: Muestra. B: Sección transversal, anillos demarcados y canal axial de resina. C: Anillo con leño tardío muy desarrollado. D: Detalle de sección transversal de traqueidas axiales del leño tardío. E: Puntuaciones areoladas solitarias y biseriadas. F: Campo de cruzamiento con puntuaciones pinoides. G: Traqueidas radiales con identaduras. H: Sección tangencial, radios con canal radial de resina. Barras. B, C: $100 \mu$ D, E, F, G, H: $20 \mu$ 

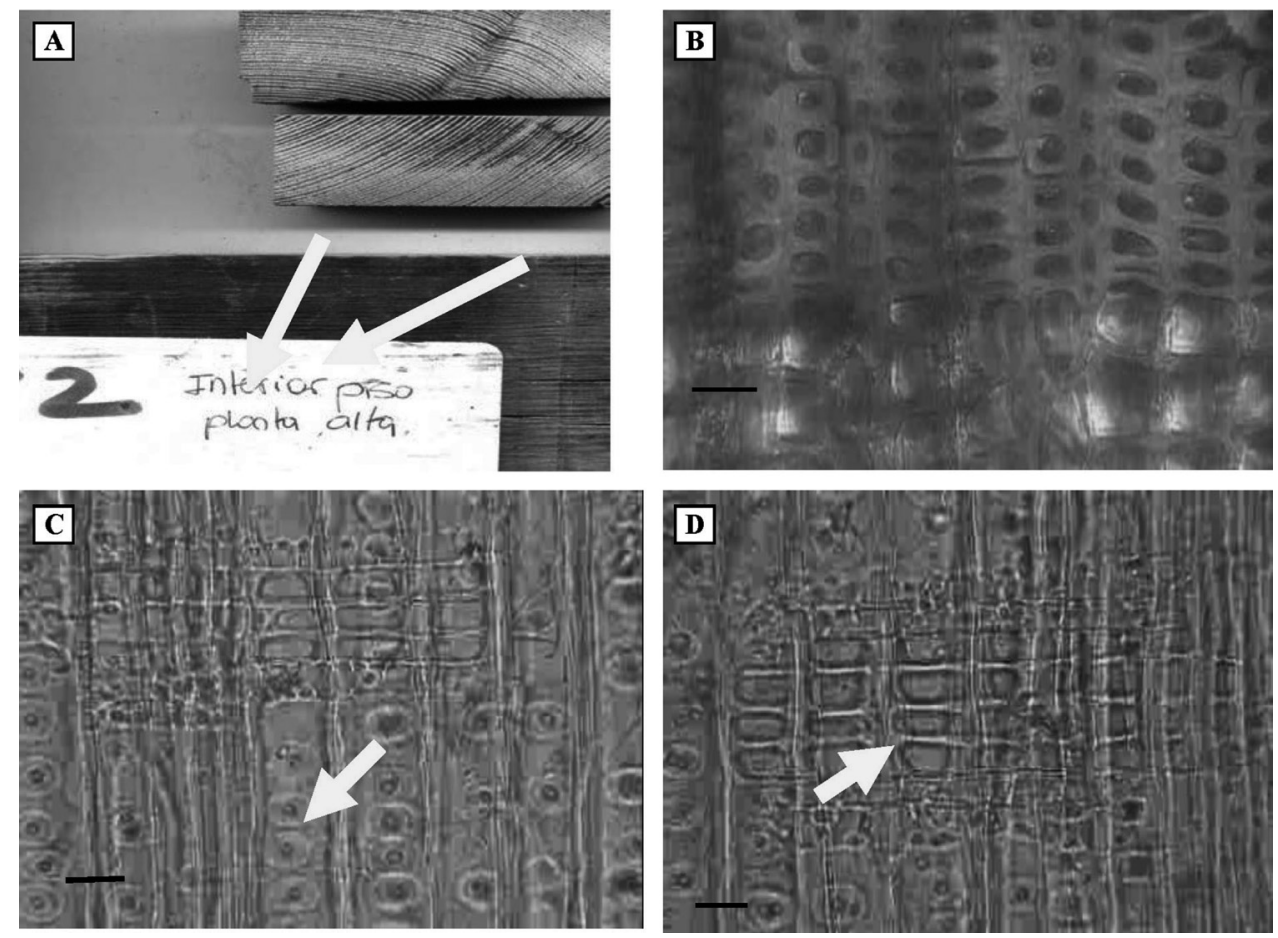

Fig. 5. Pinus sylvestris L. A: Muestra de madera. B: Sección transversal del leño, transición leño temprano/ tardío muy marcada. C: Sección radial, traqueidas con puntuaciones areoladas. D: Sección radial con puntuaciones en el campo de cruzamiento del tipo fenestriforme. Barras. B, C, D: $20 \mu$

\section{3 - Torre Céspedes (Fig. 6)}

En la Fig. 6 se indica la vista interior de la escalera de la torre (Fig. 6A) y de las muestras (Fig. 6B). La madera se encuentra en mal estado de conservación, se observa degradación del leño (Fig. 6C). Madera traqueidal, con anillos de crecimiento muy demarcados, de transición abrupta entre leño temprano y tardío. Traqueidas axiales con puntuaciones uniseriadas, se observan crásulas (Fig. 6D). Las traqueidas del leño temprano presentan diámetro medio de $37 \mathrm{u}$ y el diámetro de las puntuaciones es de $25 \mathrm{u}$. Radios exclusivamente uniseriados heterogéneos, de altura máxima 10-15 células. Puntuaciones del campo de cruzamiento fenestriformes con 1-2 por célula. Traqueidas radiales de paredes con identaduras (Fig. 6E). Ambas muestras presentan esporas de hongos (Fig. 6F), huevos de insectos (Fig. 6G) y degradación de la pared celular (Fig. $6 \mathrm{H}$ ).

M1- y M2: Gimnosperma, Familia Pinaceae, Género Pinus, especie: Pinus taeda L.
La importación de madera de pino en Argentina

Las maderas determinadas en el presente estudio, corresponden a las llamadas "pinotea". Sus características relevantes son: alto contenido de resina, diferencia muy marcada entre leño temprano y tardío, provienen de especies de crecimiento lento y árboles de grandes dimensiones. Densidad, dureza, contracción, flexión y elasticidad/flexibilidad son las características contempladas para elegir una madera para uso estructural. El pino no destaca en una de ellas pero, globalmente, es la madera que proporciona la mejor combinación (García Esteban et al., 1996).

El éxito de la permanencia en el tiempo de una estructura de madera a lo largo de cientos de años depende, entre otros factores, de la especie seleccionada. La protección preventiva de la madera engloba tanto la protección química como la correcta instalación en obra y los detalles constructivos. En función de la especie de madera elegida, en algunos casos podrían bastar medidas de tipo constructivo, y en otros será necesario 
Bol. Soc. Argent. Bot. 49 (4) 2014
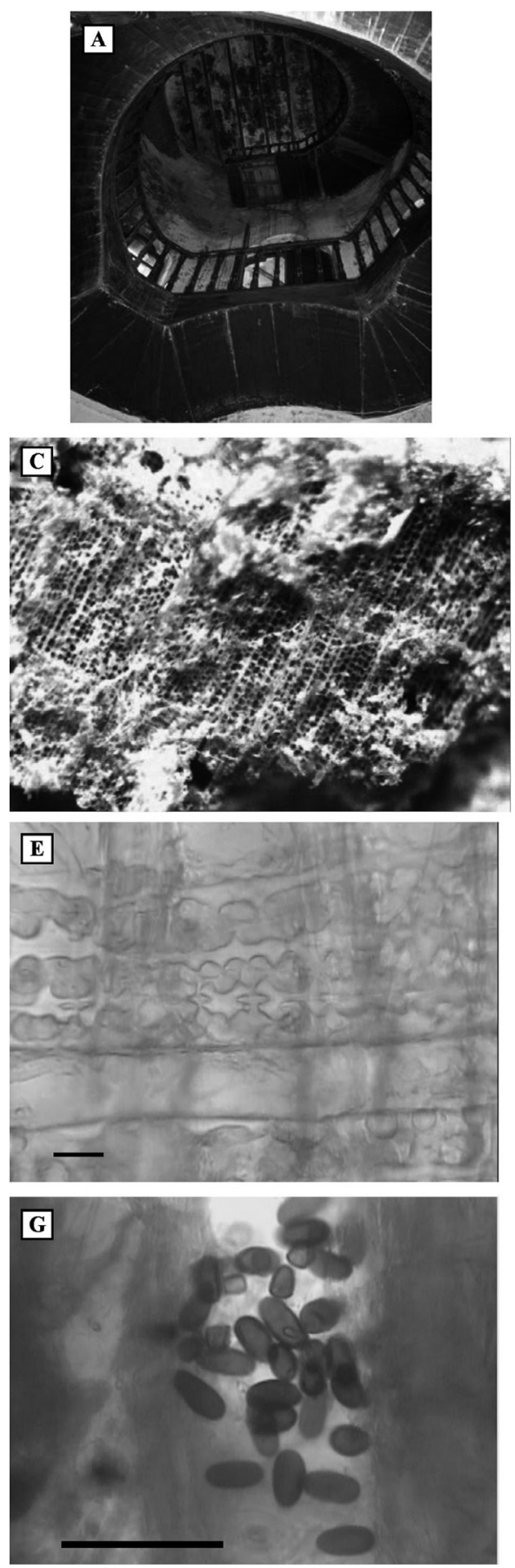
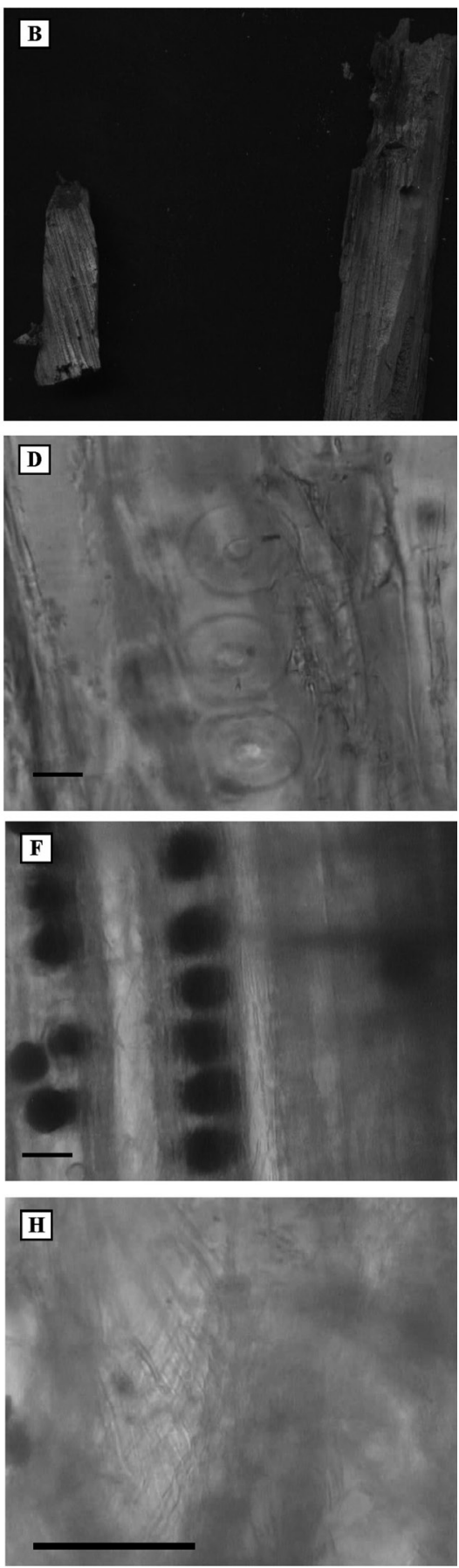

Fig. 6 Pinus taeda L. A: Vista de la escalera helicoidal desde la planta baja. B: Muestras de madera. C: Sección transversal del leño donde se observa el deterioro de la madera. D: Sección radial, traqueidas con puntuaciones areoladas con crásulas. E: Sección radial, traqueidas radiales con identaduras. F: Sección radial con esporas de hongos. G: Huevos de insectos en la madera. H: Madera degradada. Barras D, E, F, G, H: $20 \mu$ 
complementarlas con la incorporación de productos químicos. Las clases de uso intentan valorar el riesgo de ataque que pueden producir los diferentes agentes xilófagos a los elementos de madera en función del lugar donde se van a instalar. Dependen principalmente del grado de humedad que puede alcanzar la madera durante su vida de servicio y de la zona geográfica en que se instalará (AITIM, 2008).

Algunas construcciones, hoy históricas, fueron casas prefabricadas traídas de Europa con el fin de establecer de manera rápida un sitio de trabajo para alguna empresa. Este es el caso de la casa Museo Parisi. Situación similar hace referencia la Estación Hidrobiológica de Puerto Quequén que lleva ya más de 85 años de existencia, contribuyendo al conocimiento científico de las ciencias del mar en el Océano Atlántico Sudoccidental. Sus instalaciones, son en sí mismas un monumento histórico. El sencillo y viejo edificio de madera tiene interés histórico propio, y fue cedido por la empresa holandesa que construyó el Puerto de Quequén. Toda su estructura fue importada directamente desde Holanda en 1902. Sus paredes están formadas por dos tabiques de madera resinosa de pino y el espacio entre ellos estuvo primitivamente rellenado con turba, que funcionaba como un aislante excelente (Museo Bernardino Rivadavia, 2014).

A través de estudios anatómicos se puede inferir de cuán lejos se transportaba la madera (Eckstein \& Wrobel, 2006). En las tres construcciones se determinan maderas exóticas pertenecientes al género Pinus. Este cambio en el uso de las maderas nativas por otras exóticas (Giménez et al. 2014), coincide con la apertura del comercio con EEUU y Europa a partir de 1850, según está documentado para construcciones del Río de la Plata (Evans et al., 2008).

\section{Conclusiones}

Las maderas de gimnospermas analizadas fueron introducidas a la Argentina en la segunda mitad del siglo XIX y fueron empleadas en construcciones de envergadura arquitectónica.

Las técnicas anatómicas son una buena herramienta para la reconstrucción histórica de este tipo de materiales. Asimismo, a partir de estos estudios, se pueden inferir los cambios en el empleo de la madera en el país y la apertura a los mercados europeos y americanos, a partir del arribo de la pinotea.

\section{Bibliografía}

AITIM, 2008. Productos de madera para la arquitectura http://www.cscae.com/area_tecnica/aitim/ actividades/act_paginas/productos_maderea_ arquitectura.pdf.

BAUCH, J. \& D. ECKSTEIN, 1981. Wood biological investigations on panels of Rembrandt paintings. Wood Science and Technology 15: 251-263.

CARRERAS, R. \& R. DESCHAMPS. 1995. Anatomía de maderas de 157 especies forestales que crecen en Cuba y sus aspectos tecnológicos, históricos y culturales. Vol. 1-2. Teruven.

ECKSTEIN, D. \& S. WROBEL. 2006. Dendrochronological proof of origin of historic timber retrospect and perspectives. In: PROC. DENDROSYMPOSIUM 2006. Tree Rings in Archaeology, Climatology and Ecology (TRACE), 20.-22.04.2006, Tervuren/ Belgium. K. Haneca, A. Verheyden, H. Beeckman, H. Gärtner, G. Helle \& G.H. Schleser (Eds.). Jülich: Forschungszentrums Jülich 2006, S. $8-20=$ Schriften des Forschungszentrums Jülich, Reihe Umwelt/Environment. Nr. 74.

EVANS, S., M. MARCHESONI, C. MEYER \& M. CALONE. 2008. La construcción en madera en Uruguay. Una historia en el tintero. 89 pags. Tesis. http://www.farq.edu.uy/tesinas/wpcontent/blogs.dir/220/files/2012/09/Tesina-LaConstrucci\%C3\%B3n-en-Madera-en-Uruguay.pdf.

FREUND, H. 1970. Handbuch der Mikroscopie in der Techknik. Frankfurt: Umschan Verlag.

GARCÍAESTEBAN, L., A. GUINDEO \& P. PALACIOS. 1996. Maderas de Coniferas: Anatomía de Géneros. Fundación Conde del Valle de Salazar.

GIMÉNEZ, A.M. \& J.C. MOGLIA. 1999. Anatomía de Maderas. Serie Técnica 1. FCF. UNSE.

GIMÉNEZ, A.M., J.G. MOGLIA \& J. GÓMEZ. 2012. Maderas empleadas en las primeras construcciones históricas jesuíticas de Córdoba, Argentina. Cd III Congreso Iberoamericano de protección de la Madera, Ciudad Concepción, Chile.

GIMÉNEZ, A.M., J.G. MOGLIA \&, J. GÓMEZ. 2014. ¿Cómo documentar el ingreso de la madera de pino en Argentina a partir de construcciones históricas? 16as Jornadas Técnicas Forestales y Ambientales Misiones. CD. 8 pág. Mayo 2014.

GÓMEZ, J L. \& M. RUATA. 2009. Evaluación de la seguridad de un edificio singular de interés patrimonial con manifestaciones patológicas 
CONPAT 2009. X Congreso Latinoamericano de Patología y XII Congreso de Calidad en la Construcción. P: 1-5.

GREGUSS, P. 1955. Identification of living gymnosperms on the basis of xylotomy. Akadémiai Kiadó, Budapest.

JACQUIOT, C. 1955. Atlas D ánatomie des Bois Des confieres. Paris. Centre Technique du bois. Paris, Francia.

JOHNSON, H. 1989. La Madera. Editorial Blume, Barcelona.

MANTILLA CARRASCO, E., A. ROCHA TEIXEIRA, A. PIRES AZEVEDO \& L. MOREIRA. 2009. Metodología para análisis de la integridad de estructuras históricas de madera atacadas por insectos xilófagos por medio del ultrasonido. Kurú: Revista Forestal (Costa Rica) 6(16): 1-9.

MUSEO BERNARDINO RIVADAVIA. 2014. Estación Hidrobiológica de Puerto Quequén. http://www. macn.secyt.gov.ar/elmuseo/ehpq/historia.php.

RICHTER, H. G.; GROSSER, D.; HEINZ, I. \& P. GASSON. 2004. IAWA list of microscopic features for softwood identification. IAWA Journal 25 (1): $1-70$.
RODRÍGUEZ TROBAJ, E. 2008. Procedencia y uso de madera de pino silvestre y pino laricio en edificios históricos de Castilla y Andalucía. España. Arqueología de la Arquitectura 5: 33-53.

ROMERO, A. 2014. El consumo de madera como un bien ambiental. AEIM Noticias PEFC 47: p. 3.

SEC \& SPC. 2001. Guía de Coníferas de Estados Unidos. 14 p. Ed. Royaume-Uni. http://www.softwood. org/cms/data/img/uploads/files/SEC_Guide_ Softwoods_ES.pdf.

SECRETARÍA- DE TURISMO. 2014. http://www. interpatagonia.com/paseos/museopioneros/.

TORTORELLI, L. 2009. Maderas y Bosques argentinos. $2^{\mathrm{a}}$ ed. Tomo I y II. Buenos Aires, Orientación Gráfica Editora.

Recibido el 4 de julio de 2014, aceptado el 10 de septiembre de 2014. 